\title{
A new score system for predicting response to cardiac resynchronization therapy
}

\author{
Yu Kang ${ }^{1,2 *}$, Leilei Cheng ${ }^{2 *}$, Jie $\mathrm{Cui}^{3}$, Lin $\mathrm{Li}^{2}$, Shengmei Qin ${ }^{3}$, Yangang $\mathrm{Su}^{3}$, Jialiang Mao \\ Xue Gong ${ }^{2}$, Haiyan Chen ${ }^{2}$, Cuizhen Pan ${ }^{2}$, Xuedong Shen ${ }^{1}$, Ben He ${ }^{1}$, Xianhong Shu ${ }^{2}$ \\ ${ }^{1}$ Department of Cardiology, Renji Hospital, School of Medicine, Shanghai Jiaotong University, Shanghai, China \\ ${ }^{2}$ Department of Echocardiography, Zhongshan Hospital of Fudan University, \\ Shanghai Institute of Cardiovascular Disease, Shanghai, China \\ ${ }^{3}$ Department of Cardiology, Zhongshan Hospital of Fudan University, \\ Shanghai Institute of Cardiovascular Disease, Shanghai, China
}

\begin{abstract}
Background: The aim of this study was to establish a score system derived from clinical, echocardiographic and electrocardiographic indexes and evaluate its clinical value for cardiac resynchronization therapy (CRT) patient selection.

Methods: Ninety-three patients receiving CRT were enrolled. A patient selection score system was generated by the clinical, echocardiographic and electrocardiographic parameters achieving a significant level by univariate and multivariate Cox regression model. The positive response to CRT was a left ventricular end systolic volume decrease of $\geq 15 \%$ and not reaching primary clinical endpoint (death or re-hospitalization for heart failure) at the end of follow-up.

Results: Thirty-nine patients were CRT non-responders (41.94\%) and 54 were responders (58.06\%). A 4-point score system was generated based on tricuspid annular plane systolic excursion (TAPSE), longitudinal strain (LS), and complete left bundle branch block (CLBBB) combined with a wide $Q R S$ duration $(Q R S d)$. The sensitivity and specificity for prediction of a positive response to CRT at a score $>2$ were 0.823 and 0.850, respectively (AUC: $0.92295 \%$ CI 0.691-0.916, $p<0.001$ ).

Conclusions: A patient selection score system based on the integration of TAPSE, LS and $C L B B B$ combined with a wide QRSd can help to predict positive response to CRT effectively and reliably. (Cardiol J 2015; 22, 2: 179-187)
\end{abstract}

Key words: two-dimensional speckle tracking echocardiography, real-time three-dimensional echocardiography, cardiac resynchronization therapy

\section{Introduction}

Cardiac resynchronization therapy (CRT) offers proven benefit to patients with heart failure of wide QRS duration (QRSd). Although several large randomized clinical trials have demonstrated improved symptoms, reduced hospital admissions and prolonged survival, up to $30 \%$ of these carefully selected patients do not benefit from this invasive and costly intervention $[1,2]$.

Address for correspondence: Xianhong Shu, PhD, Department of Echocardiography, Zhongshan Hospital of Fudan University, Shanghai Institute of Cardiovascular Disease, No. 180 Fenglin Rd Shanghai, China, 200032, tel: +862164041990, e-mail: shu.xianhong@zs-hospital.sh.cn

AND

Ben He, PhD, Department of Cardiology, Renji Hospital, School of Medicine, Shanghai Jiaotong University, No. 1630 Dongfang Rd Shanghai, China, 200126, tel: +862168383051, e-mail: heben1025@126.com

*Yu Kang and Leilei Cheng contribute equally to the work.

Received: 11.05.2014

Accepted: 24.10.2014 
Correcting mechanical dyssynchrony within the left ventricular (LV) was proposed as one of the key mechanisms of benefit from CRT [3, 4]. However, the PROSPECT trial demonstrated that no single echocardiographic measurement of dyssynchrony may be recommended to improve patient selection for CRT [5]. It was one of the reasons that many clinical, electrocardiographic and echocardiographic features may affect CRT response.

The purpose of this study was to evaluate whether a score system integrating clinical, electrocardiographic and echocardiographic factors could help to predict CRT response.

\section{Methods}

\section{Study population}

A total of 106 dilated cardiomyopathy patients who received CRT in Zhongshan Hospital and Renji Hospital were enrolled in this study between October 2010 and September 2013. Exclusion criteria were: (1) the presence of atrial fibrillation or irregular heart rhythm, (2) poor echocardiographic image. All subjects gave their informed consent, and the local Ethics Committee approved the protocol.

\section{CRT device implantation}

The criteria for CRT implantation were: (1) LV ejection fraction (LVEF) $\leq 35 \%$, (2) QRSd $\geq 120 \mathrm{~ms}$ and (3) New York Heart Association (NYHA) functional class III or IV despite optimal pharmacological therapy. The right atrial and right ventricular (RV) leads were positioned at right atrial appendage and RV apex. The LV pacing lead was inserted in a lateral or postero-lateral vein. AV and VV delays were optimized under echocardiography after implantation to maximize aortic velocity time integral. Patients were regularly followed up at 6 months interval or when symptoms deteriorated. All patients' histories, medications, assessments of heart failure, electrocardiogram (ECG) and echocardiography were obtained before CRT implantation and during regular follow-up.

\section{Electrocardiogram}

Patients underwent 12-lead ECG at a paper speed of $25 \mathrm{~mm} / \mathrm{s}$ at baseline and during followup. The QRSd was measured using the widest QRS complex in leads II, $\mathrm{V}_{1}$ and $\mathrm{V}_{6}$. Data from at least 3 distinct cardiac cycles were averaged. QRS morphology was defined as complete left bundle branch block (CLBBB) or non-CLBBB. Experienced observers who were blind to all other patient data interpreted the ECG data.

\section{Clinical assessment}

An independent physician blinded to all other data performed the clinical evaluation. The assignment to ischemic etiology was based on clinical history of prior myocardial infarction, prior percutaneous coronary intervention, prior coronary artery bypass surgery, or the angiographic finding of $>70 \%$ stenosis of at least one major coronary artery, similar to the assignment used in previous studies [6]. The primary endpoint was unplanned hospitalization for a major cardiovascular event or death from any cause. A positive response to CRT was defined as a decrease in LV end diastolic volume $\geq 15 \%$ at the end of follow-up compared with baseline and not reach the primary endpoint at the end of follow-up after CRT.

\section{Echocardiographic imaging}

Images were obtained in the left lateral decubitus position with a commercially available system (iE33, Philips Medical Systems, N.A., Bothell, WA, USA) equipped with S5-1 and X3. Standard two-dimensional (2D) images were acquired according to recommendations of the American Society of Echocardiography [7]. For acquisitions of a full-volume data set of real-time 3-dimensional echocardiography (RT3DE), 4 smaller real-time volumes were combined to provide a larger pyramidal volume, during breath hold and required a relatively stable $\mathrm{R}-\mathrm{R}$ interval to minimize translation artifacts between the 4 acquired subvolumes. Depth was minimized to optimize the frame rate. At least 3 beats for 2D images were digitally stored for offline analysis. In this study, a frame rate of 53 to 72 FPS (frame rate per second) for speckle tracking analysis and 19 to 34 FPS for RT3DE analysis were used.

\section{Conventional echocardiographic indexes analysis}

Each measurement was taken from the average of 3 continuous cardiac cycles. The severity of mitral regurgitation (MR) and tricuspid regurgitation (TR) were analyzed and categorized into 3 grades: mild, moderate and severe by the color flow jet area, as recommended by the American Society of Echocardiography [8]. RV and LV preejection delay was determined as the time from the QRS onset to the onset of pulsed Doppler flow in the LV and RV outflow tracts. Interventricular mechanical delay (IVMD) was calculated as the difference in LV and RV pre-ejection delay. Tricuspid annular plane systolic excursion (TAPSE) was acquired by placing an M-mode cursor through 
the tricuspid annulus and measuring the amount of longitudinal motion of the annulus at peak systole. Systolic pulmonary artery pressure (SPAP) was determined from peak TR jet velocity, using the simplified Bernoulli equation and combined this value with an estimate of the right atrial pressure.

\section{Speckle tracking-derived strain value analysis}

QLAB version 8.1 (Philips Medical System, N.A., Bothell, WA, USA) was used for strain and LVEF analysis. The automatic tracking analysis was performed in the apical 4-chamber, 2-chamber, apical LV longitudinal view for longitudinal strain (LS) and in the parasternal short-axis view at basal and mid-papillary level for circumferential and radial strain (RS) according to the vendor's instructions. The endocardial border was manually traced at end-diastole. Tracking was then performed and the strain-time curves were obtained automatically. The strain-time curves were analyzed manually, and if tracking was suboptimal, the endocardial border was retraced. If satisfactory tracking was not accomplished within 3 attempts, the non-tracking segments were excluded from analysis.

Peak LS measurements were obtained from the basal, mid-segments of the anterior, inferior, anteroseptal, anterolateral, inferoseptal, inferolateral walls, apical segments of anterior, inferior, septal, lateral walls and apex, 17 segments in total. Peak circumferential strain (CS) and RS measurements were obtained from the basal, mid-segments of the anterior, inferior, anteroseptal, anterolateral, inferoseptal, inferolateral walls, 12 segments in total. Regional strain values were averaged to determine global LS/CS/RS.

Standard deviations of the time to negative peak value of LS for 17 segments (LS-SD), of the time to negative peak value of CS for 12 segments (CS-SD) and of the time to positive peak value of RS for 12 segments (RS-SD) were calculated to evaluate LV dyssynchrony. LS-SD, CS-SD and RS-SD were expressed as percentages of the duration of the cardiac cycle.

\section{RT3DE-derived LVEF analysis and systolic dyssynchronization index}

Five reference points were set by the user at the end-diastolic and the end-systolic frames separately: the septal, lateral mitral annulus at apical 4-chamber view; anterior, inferior mitral annulus at apical 2-chamber view and the apex (from either one of the views). Then the software automatically delineated the LV endocardial border and created a mathematical model that represented the LV cavity. After detection of the endocardium borders, the user can correct the shape of the LV reference at the end-diastolic or end-systolic reference frame. The intraventricular dyssynchrony of LV was evaluated by the standard deviation of the time to reach minimum regional volume for 16 segments, defining the systolic dyssynchronization index (SDI) as described by Kapetanakis et al. [9].

\section{Statistical analysis}

Continuous variables were expressed as the mean \pm standard deviation. Nominal variables were expressed as percentages. Differences between beseline and follow-up were determined using one-way ANOVA test for continuous variables and Kruskal-Wallis test for categorical data. The baseline variables analyzed were: (1) age, (2) gender, (3) hypertension, (4) diabetes mellitus, (5) etiology of heart failure (ischemic or non-ischemic), (6) NYHA class, (7) clearance of creatinine calculated by Cockcroft-Gault equation, (8) CLBBB combined with QRSd $\geq 150 \mathrm{~ms}$, (9) left ventricular end diastolic diameter (LVEDD), (10) left ventricular end systolic diameter (LVESD), (11) left ventricular end diastolic volume (LVEDV), (12) left ventricular end systolic volume (LVESV), (13) LVEF, (14) degree of MR, (15) degree of TR, (16) SPAP, (17) TAPSE, (18) IVMD, (19) SDI, (20) LS, (21) CS, (22) RS, (23) LS-SD, (24) CS-SD, and (25) RS-SD. Possible predictors of CRT response were determined by univariate Cox regression. Variables that achieved a significant level of $\mathrm{p}<0.1$ were selected for evaluation by a multivariate Cox regression model. Score system was generated by the variables with a significant level of $\mathrm{p} \leq 0.05$ by multivariate Cox regression. The Wald test was used to evaluate the weight of the variables to predict CRT response. We assigned 2 points for the variable with the highest value of Wald. Higher scores reflected a greater likelihood to respond to CRT. A receiver operating characteristic curve (ROC) was obtained and the optimal value with the greatest total of sensitivity and specificity in the prediction of CRT response was selected. KaplanMeier plots were performed for each parameter used. Data were analyzed by SPSS version 16.0 (SPSS, Inc, Chicago, IL, USA). A value of $\mathrm{p} \leq 0.05$ was considered significant.

\section{Results}

One patient was not implanted with CRT successfully because of coronary sinus anatomy. Six 
Table 1. Baseline characteristics of patients.

\begin{tabular}{lcccc}
\hline & All patients & Responders & Non-responders & Deaths \\
\hline $\mathrm{N}$ & 93 & 54 & 39 & 16 \\
Age [years] & $60.8 \pm 12.7$ & $60.8 \pm 12.2$ & $60.7 \pm 13.5$ & $66.0 \pm 14.2$ \\
Male & $66(70.97 \%)$ & $31(57.41 \%)$ & $28(71.79 \%)$ & $11(68.75 \%)$ \\
Hypertension & $33(35.48 \%)$ & $24(44.44 \%)$ & $9(23.08 \%)$ & $5(31.25 \%)$ \\
Diabetes mellitus & $15(16.13 \%)$ & $13(24.07 \%)$ & $2(5.13 \%)$ & $1(6.25 \%)$ \\
Valvular disease & $2(2.15 \%)$ & $1(1.85 \%)$ & $1(2.56 \%)$ & $0(0.00 \%)$ \\
Ischemic cardiomyopathy & $16(17.20 \%)$ & $9(16.67 \%)$ & $7(17.95 \%)$ & $4(25.00 \%)$ \\
CLBBB & $42(45.16 \%)$ & $33(61.11 \%)$ & $9(23.08 \%)$ & $5(31.25)$ \\
ACEl/ARB & $80(86.02 \%)$ & $47(87.04 \%)$ & $33(84.62 \%)$ & $14(87.50 \%)$ \\
Beta-blocker & $83(89.25 \%)$ & $51(94.44 \%)$ & $32(82.05 \%)$ & $14(87.50 \%)$ \\
Loop diuretics & $74(79.57 \%)$ & $47(87.04 \%)$ & $27(69.23 \%)$ & $13(81.25 \%)$ \\
Spironolactone & $67(72.04 \%)$ & $42(77.78 \%)$ & $25(64.10 \%)$ & $14(87.50 \%)$ \\
Digoxin & $47(50.54 \%)$ & $28(51.85 \%)$ & $19(48.72 \%)$ & $11(68.75 \%)$ \\
\hline
\end{tabular}

ACEI — angiotensin-converting enzyme inhibitor; ARB — angiotensin receptor blocker; CLBBB — complete left bundle branch block

patients were lost during follow-up. After exclusion of 3 patients due to poor image and 3 due to atrial fibrillation, the remaining 93 patients formed the final study group. The baseline characteristics of the study population were listed in Table 1. Optimal medical therapy for heart failure was given to all patients prior to and after CRT implantation. The follow-up duration was $20.1 \pm 10.7$ months, ranging from 3 to 42 months. The following primary endpoints of cardiovascular events occurred in $20(21.51 \%)$ patients: $16(17.20 \%)$ deaths from worsening heart failure and $4(4.44 \%)$ hospitalizations for deteriorating heart failure. The follow-up duration was $6.8 \pm 4.6$ months, ranging from 4 to 21 months for those deaths. A total of $39(41.94 \%)$ patients were non-responders and 54 (58.06\%) were responders.

\section{Changes of echocardiographic values}

At the end of follow-up, in all patients, QRS duration decreased from $167.25 \pm 30.94 \mathrm{~ms}$ to $142.00 \pm$ $\pm 23.21 \mathrm{~ms}(\mathrm{p}=0.000)$. LVEDV and LVESV decreased significantly, which resulted in an increase of LVEF at follow-up. A significant improvement of intra- and inter-ventricular dyssynchrony was demonstrated. SPAP improved from $44.29 \pm 17.39 \mathrm{~mm}$ $\mathrm{Hg}$ to $36.61 \pm 15.18 \mathrm{~mm} \mathrm{Hg}(\mathrm{p}=0.000)$. However, all these values showed no significant improvement in non-responders. In all patients, no difference of LS, CS, RS and TAPSE was observed after CRT improvement. However, in responders, LS showed the tendency of improvement (from $-8.52 \pm 2.10 \%$ to $-9.09 \pm 2.96 \%, p=0.051$ ) at the mid-term of follow-up, and improved significantly at the end of follow-up (to $-9.54 \pm 3.64 \%, \mathrm{p}=0.017$ ) (Table 2).

\section{Predictors of CRT response}

The variables of CLBBB combined with a wide QRSd, TAPSE, SDI, LS and CS achieved a significant level of $p<0.1$ by univariate Cox regression when dependent variable was a positive response to CRT (Table 3). However, in multivariate Cox regression analysis, only TAPSE remained significant in predicting CRT response (HR: 1.095, Wald value: $7.570, \mathrm{p}=0.006,95 \% \mathrm{CI}: 1.026-1.168$ ) (Table 3). Table 4 listed the results of univariate Cox regression with 4 variables with a $\mathrm{p}<0.1$ when the dependent variable was mortality. In multivariate Cox regression analysis, LS (HR: 1.398, Wald value: $5.355, p=0.006,95 \% \mathrm{CI}: 1.053-1.858)$ and CLBBB with a wide QRSd (HR: 5.360, Wald value: 4.161, $\mathrm{p}=0.041,95 \%$ CI: 0.037-0.936) achieved a significant level. The cutoff points for TAPSE and LS to predict positive response of CRT were calculated from ROC curves (Table 5, Fig. 1).

A 4-point score was generated based on the variable significant by multivariate Cox regression when the dependent variable was response to CRT and on the 2 variables significant when the dependent variable was death. We assigned 1 point each for CLBBB combined with a wide QRSd, and LS $\leq$ $-7.22 \%$, and 2 points for TAPSE $\geq 14.8 \mathrm{~mm}$.

The overall mean score was $2.38 \pm 1.19$. The mean score was $3.08 \pm 0.85$ in responders, $1.44 \pm$ \pm 0.91 in non-responders and $0.81 \pm 0.98$ in deaths. The cutoff point for score system was $>2$ by ROC analysis (Fig. 2). CRT responders, non-responders and deaths with a score $>2$ were $50 / 54(92.59 \%), 11 / 39(28.21 \%)$ and $4 / 16(25.00 \%)$, respectively (Table 6 ). The score system was 


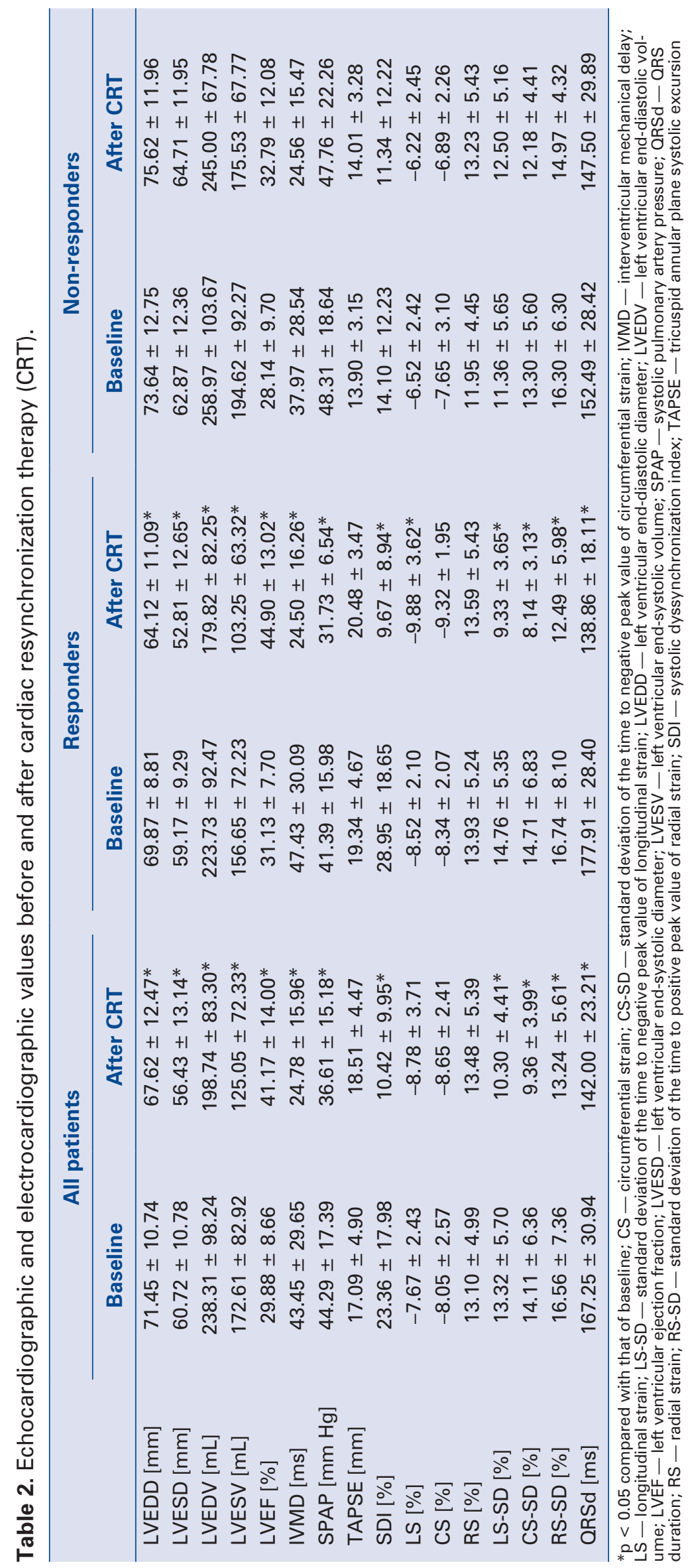


Table 3. Univariate Cox regression for variables related to cardiac resynchronization therapy response.

\begin{tabular}{lcccc}
\hline Variables & Hazard ratios & Wald value & P & $95 \% \mathbf{C l}$ \\
\hline CLBBB with a wide ORSd & 2.555 & 5.273 & 0.022 & $1.147-5.690$ \\
Longitudinal strain & 0.861 & 5.191 & 0.023 & $0.756-0.979$ \\
Circumferential strain & 1.123 & 3.761 & 0.052 & $0.999-1.264$ \\
TAPSE & 1.087 & 6.711 & 0.010 & $1.020-1.157$ \\
SDI & 1.016 & 5.289 & 0.021 & $1.002-1.029$ \\
\hline
\end{tabular}

$\mathrm{Cl}$ - confidential interval; CLBBB — complete left bundle branch block; QRSd — QRS duration; TAPSE — tricuspid annular plane systolic excursion; $\mathrm{SDI}$ - systolic dyssynchronization index

Table 4. Univariate Cox regression for variables related to deaths.

\begin{tabular}{lcccc}
\hline Variables & Hazard ratios & Wald value & $\mathbf{P}$ & $\mathbf{9 5 \%} \mathbf{C l}$ \\
\hline TAPSE & 0.749 & 10.178 & 0.001 & $0.628-0.895$ \\
Mitral regurgitation & 2.079 & 4.110 & 0.043 & $1.025-4.219$ \\
Longitudinal strain & 1.549 & 11.881 & 0.001 & $1.208-1.986$ \\
CLBBB with a wide ORSd & 0.088 & 10.269 & 0.001 & $0.020-0.390$ \\
\hline
\end{tabular}

$\mathrm{Cl}$ — confidential interval; CLBBB — complete left bundle branch block; QRSd — QRS duration; TAPSE — tricuspid annular plane systolic excursion

Table 5. Cutoff point, sensitivity and specificity of tricuspid annular plane systolic excursion (TAPSE), dyssynchronization index, longitudinal strain (LS) and QRS duration (QRSd).

\begin{tabular}{lcccccc}
\hline & AUC & P & Cut-off point & Sensitivity & Specificity & 95\% Cl \\
\hline TAPSE & 0.840 & 0.000 & $\geq 14.8 \mathrm{~mm}$ & 0.880 & 0.722 & $0.744-0.912$ \\
CLBBB with wide QRSd & 0.837 & 0.000 & - & 0.868 & 0.821 & $0.740-0.909$ \\
LS & 0.785 & 0.000 & $\leq-7.22 \%$ & 0.804 & 0.737 & $0.681-0.818$ \\
\hline
\end{tabular}

AUC — area under curve; $\mathrm{Cl}$ — confidential interval; CLBBB — complete left bundle branch block

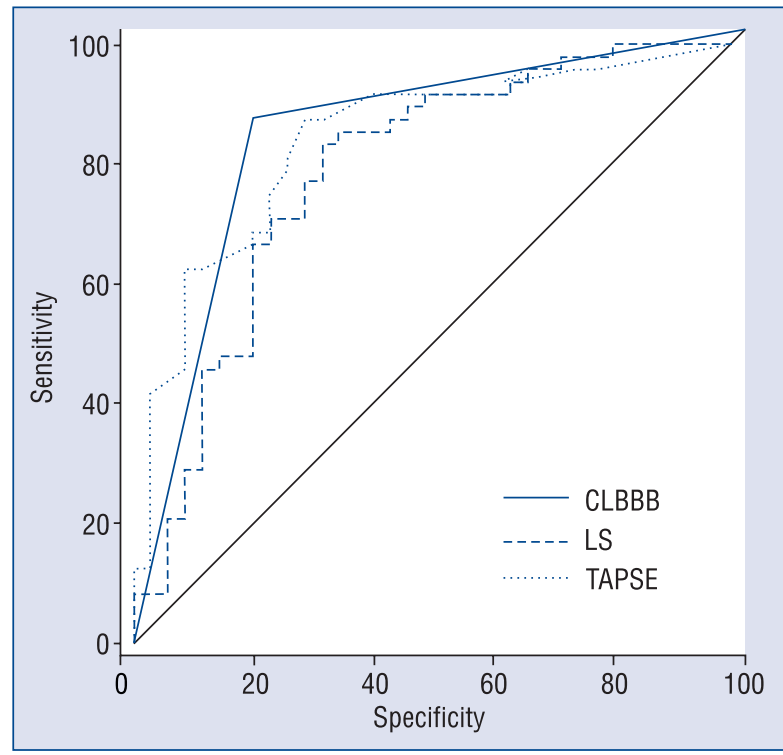

Figure 1. Receiver operating characteristic curves for 3 variables in the prediction of cardiac resynchronization therapy response; CLBBB - complete left bundle branch block; LS - longitudinal strain; TAPSE — tricuspid annular plane systolic excursion.

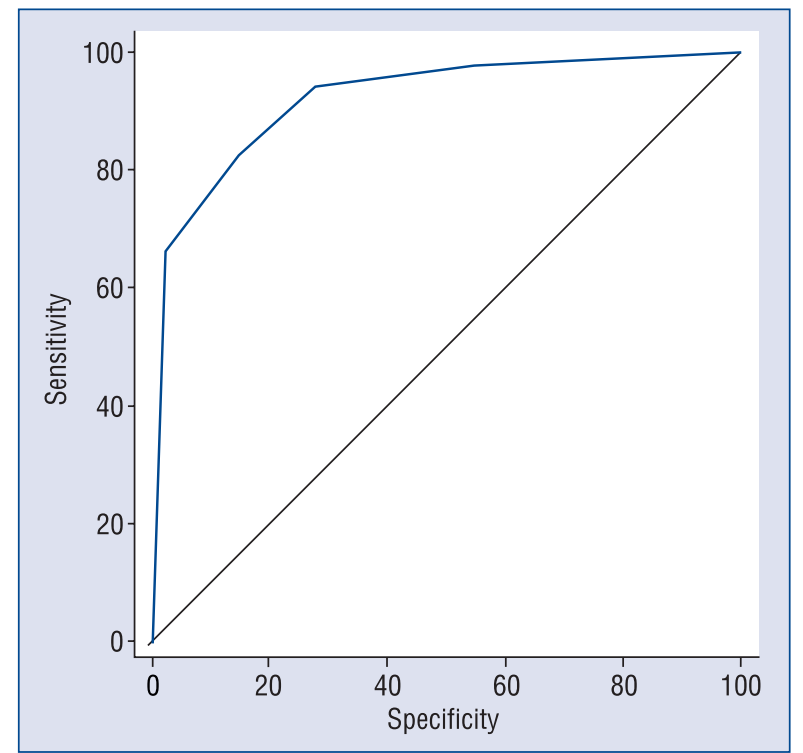

Figure 2. Receiver operating characteristic curve for score system in the prediction of cardiac resynchronization therapy response (cutoff point: $>2$; AUC: 0.922; sensitivity: 0.823 ; specificity: $0.850 ; p<0.001 ; 95 \%$ Cl: 0.691-0.916). 
Table 6. Sensitivity, specificity, negative predictive value (NPV) and positive predictive value (PPV) of score system to predict cardiac resynchronization therapy response.

\begin{tabular}{lcccc}
\hline & Sensitivity & Specificity & PPV & NPV \\
\hline Score $>2$ & $50 / 54(92.59 \%)$ & $28 / 39(71.79 \%)$ & $50 / 61(81.96 \%)$ & $28 / 32(87.50 \%)$ \\
\hline
\end{tabular}

associated with CRT response (HR: 5.69, $\mathrm{p}=0.000$, 95\% CI: 2.96-10.68) by Cox regression.

\section{Discussion}

Our study demonstrated that CLBBB combined with a wide QRSd, TAPSE and LS was related to CRT response. This score system could help to select patients with a higher likelihood of CRT response.

The PROSPECT trial negated the role of a single echocardiographic measurement of dyssynchrony in patient selection for CRT [5]. The EchoCRT trial also concluded that mechanical dyssynchrony assessed by tissue Doppler imaging and speckle tracking radial strain was not the primary determinant of response to CRT [10]. Furthermore, the use of CRT did not reduce the rate of death from any cause or first hospitalization for heart failure among patients with a duration of less than $130 \mathrm{~ms}$. Similarly, in our study, parameters of mechanical dyssynchrony did not help to predict response of CRT. However, CLBBB with a wide QRSd was a predictor of responder of CRT.

One of the key mechanisms of benefit from CRT is to correct mechanical dyssynchrony [3, 4]. However, in order to restore mechanical dyssynchrony, dyssynchronous segments must be viable in order to recruit their "wasted work" toward cardiac output. Patients with significant scar burden may not respond to CRT because myocardial segments are scarred and thus lack residual contractility that can be recruited by CRT [11]. In those with severe LV dysfunction, the percent of patients with detectable scar by Cardiac magnetic resonance (CMR) imaging ranged $12-100 \%$, depending on the underlying cause of heart failure [12]. Both White et al. [13] and Ypenburg et al. [14] reported that total scar burden, determined by CMR is linearly related to relative changes in LVESV after 6 months of CRT, thus the greater amount of viable myocardium present, the larger reverse remodeling. Speckle tracking imaging, which is an angle independent approach to strain analysis, has enabled the development of new parameters to assess both global and segmental function [15]. Longitudinal mechanics reflect the function of longitudinally aligned fibers within the subendocardium, the most sensitive layer to the presence of myocardial disease. After evaluating 90 patients with chronic ischemic LV dysfunction, Roes et al. [16] demonstrated that global and regional LS measured with speckle tracking was associated with the global and regional extent of scar tissue on CMR. After analyzing 1,077 ischemic and non-ischemic patients implanted with CRT, MADIT-CRT trial found that dyssynchrony and global LS were independent factors predicting CRT response and the benefit from CRT was greatest in patients with greater baseline contractile function (global LS $\leq-8.7 \%$ ) [17]. In the present study, we also found that global LS was related to CRT response.

Shen et al. [18] generated a 7-point patient selection score system based on RV pacing-induced left bundle branch block, use of beta-blockers, wall motion score index, left atrial volume index, clearance of creatinine and LV dyssynchrony analyzed by tissue Doppler imaging combined with pulsewave Doppler, to predict CRT response. However, that system did not select the RV function as variable. RV ejection fraction was correlated well with LV function [19]. Impairment of RV systolic function has been consistently associated with worse outcomes in patients with advanced heart failure, independently of LV function [20]. Previous studies reported that RV dysfunction represented a strong predictor of lack of clinical response to CRT [21-23]. In the present study, we used TAPSE, a simple, less dependent on optimal image quality and reproducible method to assess RV function and found that TAPSE was a strong variable to predict CRT response.

The hazard ratio was influenced by the units of the variables in continuous data. That was the result why we used Wald value to evaluate the weight of the variables. Therefore, we assigned 2 points to the variable reaching the highest Wald values and 1 point to other variables.

This selection score system, integrating electrical dyssynchrony, myocardial contractile function and RV function, was a strong predictor of CRT response. A score $>2$ was associated with a 6 -fold increase in CRT response compared to a score $\leq 2$. 
This score system might be helpful to target patients who could benefit from CRT implantation.

MADIT-CRT [24] trial observed that after CRT implanted for 1 year, LV dyssynchrony, which was determined as standard deviation of time to peak transverse strain, and contractile function, which was determined as global LS, improved to a great extent. We observed that after CRT implantation, inter and intraventricular dyssynchrony improved significantly. In CRT responders, LV global longitudinal improved gradually after CRT implantation and reached statistical significance at the end of follow-up. One of the key mechanisms of CRT was to correct mechanical dyssynchrony within the LV and to recruit "wasted work" of dyssynchronous segments toward cardiac output, whose hemodynamic benefit could been observed immediately after activating biventricular pacing [25]. After long-term CRT, ion channel remodeling and abnormal $\mathrm{Ca}^{2+}$ homeostasis was restored, contributing to beneficial reverse remodeling and improvement of contractile function [26]. This could partly explain the results of the present study that inter and intraventricular dyssynchrony was greatly improved at the early stage of follow-up, while strain values increased gradually.

\section{Limitations of the study}

This study included a relatively small number of patients. Not including patients with atrial fibrillation, our results should only be applied to patients with sinus rhythm. Furthermore, this score system has not been tested prospectively to determine its use in a large number of patients receiving CRT. Prospective studies with large number of patients, longer periods of follow-up with hard clinical endpoints will be necessary to determine the clinical significance of our findings in predicting CRT response.

\section{Conclusions}

A patient selection score system based on the integration of TAPSE, LS and CLBBB combined with a wide QRSd could help to predict positive response to CRT effectively and reliably.

\section{Acknowledgements}

This work was supported by grants from the National Nature Science Foundation of China (30972812, 81401411), Nature Science Foundation of Shanghai (14zr1425200) Project of Shanghai Science and Technology Commission (124119a7702).

\section{Conflict of interest: None declared}

\section{References}

1. Abraham WT, Fisher WG, Smith AL et al; MIRACLE Study Group. Multicenter InSync Randomized Clinical Evaluation. Cardiac cardiac resynchronization in chronic heart failure. $\mathrm{N}$ Engl J Med, 2002; 346: 1845-1853.

2. Cleland JG, Daubert JC, Erdmann E et al; Cardiac Resynchronization-Heart Failure (CARE-HF) Study Investigators. The effect of cardiac resynchronization on morbidity and mortality in heart failure. N Engl J Med, 2005; 352: 1539-1549.

3. Kass DA. pathobiology of cardiac dyssynchrony and resynchronization. Heart Rhythm, 2009; 6: 1660-1665.

4. Aiba T, Hesketh GG, Barth AS et al. Electrophysiological consequences of dyssynchronous heart failure and its restoration by resynchronization therapy. Circulation, 2009; 119: 1220-1230.

5. Chung ES, Leo AR, Tavazzi L et al. Results of the Predictors of Response to CRT (PROSPECT) trial. Circulation, 2008; 117: 2608-2616.

6. Bristow MR, Saxon LA, Boehmer J et al.; Comparison of Medical Therapy, Pacing, and Defibrillation in Heart Failure (COMPANION) Investigators. Cardiac-resynchronization therapy with or without an implantable defibrillator in advanced chronic heart failure. N Engl J Med, 2004; 350: 2140-2150.

7. Lang RM, Bierig M, Devereux RB et al.; American Society of Echocardiography's Nomenclature and Standards Committee; Task Force on Chamber Quantification; American College of Cardiology Echocardiography Committee; American Heart Association; European Association of Echocardiography, European Society of Cardiology. Recommendations for chamber quantification. Eur J Echocardiogr, 2006; 7: 79-108.

8. Zoghbi WA, Enriquez-Sarano M, Foster E et al.; American Society of Echocardiography. Recommendations for evaluation of the severity of native valvular regurgitation with two-dimensional and Doppler echocardiography. J Am Soc Echocardiogr, 2003; 16: $777-802$.

9. Kapetanakis S, Kearney MT, Siva A, Gall N, Cooklin M, Monaghan MJ. Real-time three-dimensional echocardiography: A novel technique to quantify global left ventricular mechanical dyssynchrony. Circulation, 2005; 112: 992-1000.

10. Ruschitzka F, Abraham WT, Singh JP et al.; EchoCRT Study Group. Cardiac-resynchronization therapy in heart failure with a narrow QRS complex. N Engl J Med, 2013; 369: 1395-1405.

11. Khan FZ, Virdee MS, Read PA et al. Effect of low-amplitude two-dimensional radial strain at left ventricular pacing sites on response to cardiac resynchronization therapy. J Am Soc Echocardiogr, 2010; 23: 1168-1176.

12. Kim RJ, Wu E, Rafael A et al. The use of contrast-enhanced magnetic resonance imaging to identify reversible myocardial dysfunction. N Engl J Med, 2000; 343: 1445-1453.

13. White JA, Yee R, Juan X et al. Delayed enhancement magnetic resonance imaging predicts response to cardiac resynchronization therapy in patients with intraventricular dyssyncrony. J Am Coll Cardiol, 2006; 21: 1953-1960.

14. Ypengurg C, Roes SD, Bleeker GB et al. Effect of total scar burden on contrast-enhanced magnetic resonance imaging on response to cardiac resynchronization therapy. Am J Cardiol, 2007; 99: 657-660.

15. Geyer H, Caracciolo G, Abe $\mathrm{H}$ et al. Assessment of myocardial mechanics using speckle tracking echocardiography: Fundamentals and clinical application. J Am Soc Echocardiogr, 2010; 23: 351-369. 
16. Roes SD, Mollema SA, Lamb HJ, van der Wall EE, de Roos A, Bax JJ. Validation of echocardiographic two-dimensional speckle tracking longitudinal strain imaging for viability assessment in patients with chronic ischemic left ventricular dysfunction and comparison with contrast-enhanced magnetic resonance imaging. Am J Cardiol, 2009; 104: 312-317.

17. Knappe D, Pouleur AC, Shah AM et al.; for the Multicenter Automatic Defibrillator Implantation Trial-Cardiac Resynchronization Therapy Investigator. Dyssynchrony, contractile function, and response to cardiac resynchronization therapy. Circ Heart Fail, 2011; 4: 433-440.

18. Shen XD, Nair CK, Aronow WS et al. A patient selection score for cardiac resynchronization therapy. Echocardiography, 2011; 28: 188-195.

19. Puwanant S, Priester TC, Mookadam F, Bruce CJ, Redfield MM, Chandrasekaran K. Right ventricular function in patients with preserved and reduced ejection fraction heart failure. Eur J Echocardiogr, 2009; 10: 733-737.

20. Abramson SV, Burke JF, Kelly JJ Jr et al. Pulmonary hypertension predicts mortality and morbidity in patients with dilated cardiomyopathy. Ann Intern Med, 1992; 10: 733-737.

21. Meyer P, Filippatos GS, Ahmed MI et al. Effects of right ventricular ejection fraction on outomes in chronic systolic heart failure. Circulation, 2012; 121: 252-258.
22. Tabereaux PB, Doppalapudi H, Kay GN, McElderry HT, Plumb VJ, Epstein AE. Limited response to cardiac resynchronization therapy in patients with concomitant right ventricular dysfunction. J Cardiovasc Electrophysiol, 2010; 21: 431-435.

23. Alpendurada F, Guha K, Sharma R et al. Right ventricular dysfunction is a predictor of non-response and clinical outcome following cardiac resynchronization therapy. Cardiovasc Magn Reson, 2011; 31: 68 .

24. Pouleur AC, Knappe D, Shah AM et al.; for the MADIT-CRT Investigator. Relationship between improvement in left ventricular dyssynchrony and contractile function and clinical outcome with cardiac resynchronization therapy: The MADIT-CRT trial. Eur Heart J, 2011; 32: 1720-1729.

25. Breithardt OA, Stellbrink C, Kramer AP et al.; PATH-CHF Study Group. Pacing Therapies for Congestive Heart Failure. Echocardiographic quantification of left ventricular asynchrony predicts an acute hemodynamic. J Am Coll Cardiol, 2002; 40: 536-545.

26. Aiba T, Hesketh GG, Barth AS et al. Electrophysiological consequences of dyssynchronous heart failure and its restoration by resynchronization therapy. Circulation, 2009; 119: $1220 \mathrm{e} 30$. 